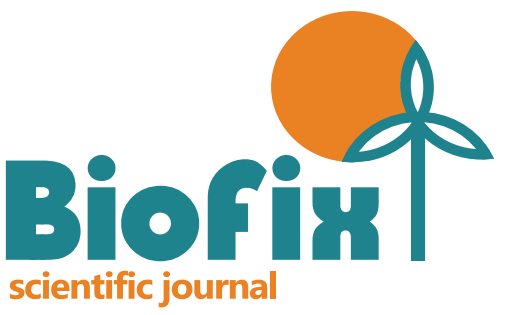

v. 2 n. 22017

Recebido em 25/07/2017

Aceito em 05/09/2017

Publicado em 11/09/2017

DOI: dx.doi.org/10.5380/biofix.v2i2.54124

\section{ESTIMATIVA DA ALTURA E DO VOLUME EM POVOAMENTOS JOVENS DE RESTAURAÇÃO FLORESTAL EM RONDÔNIA}

\author{
HEIGHT-DIAMETER AND VOLUME EQUATIONS FOR TREES IN YOUNG \\ STANDS OF RESTORATION FOREST IN RONDÔNIA
} Universidade Federal do Paraná, Curitiba, Paraná, Brasil
carlos_sanquetta@hotmail.com ${ }^{1}$, mateus.sanquetta@gmail.com ${ }^{2}$ \& anapaulacorte@gmail.com ${ }^{5}$

Carlos Roberto Sanquetta ${ }^{1}$ Mateus Niroh Inoue Sanquetta ${ }^{2}$

Alexis Bastos ${ }^{3}$

Alexandre Queiroz ${ }^{4}$ Ana Paula Dalla Corte ${ }^{5}$

Centro de Estudos Rioterra, Porto Velho, Rondônia, Brasil alexis@rioterra.org.br ${ }^{3}$ \& alexandrequeiroz@rioterra.org.br ${ }^{4}$

\section{RESUMO}

Modelos hipsométricos e volumétricos são ferramentas úteis para monitorar o desenvolvimento de plantios de restauração florestal. Neste estudo, foram ajustadas equações de estimativa de altura total $(h)$ e volume total com casca $(v)$ em função do diâmetro à altura do peito. Os dados foram coletados em 20 árvores mensuradas e cubadas em povoamentos mistos jovens de restauração, situados nos municípios de Itapuã do Oeste e Cujubim, ao Norte do estado de Rondônia, em que cinco modelos de regressão hipsométricos e volumétricos foram testados. Além disso, foi analisada a utilização de um fator de forma médio nas estimativas de volume. Os modelos foram julgados pelo coeficiente de determinação $\left(R^{2}\right)$, erro padrão da estimativa em percentagem (Syx\%) e análise gráfica dos resíduos. Todos os modelos hipsométricos apresentaram resultados satisfatórios, com Syx de $15 \%$ e $R^{2}$ variando entre 0,5443 e 0,5946, sendo o modelo de Henriksen selecionado como o melhor. Os modelos volumétricos apresentaram valores Syx da ordem de $11 \%$ e $\mathrm{R}^{2}$ variando entre 0,8497 e 0,9117 , em que o melhor modelo volumétrico foi o de Kopezky \& Gehrardt. Observou-se que existe forte correlação entre as variáveis dendrométricas analisadas (entre 0,7573 e 0,9531), bem como, que o fator de forma não foi preciso para estimar volume. Adicionalmente, os modelos de regressão, tanto hipsométricos como volumétricos, são precisos e podem gerar estimativas confiáveis para o monitoramento de povoamentos jovens de restauração na Amazônia.

PALAVRAS-CHAVE: Cubagem, Plantios jovens, Quantificação volumétrica.

\section{ABSTRACT}

Height-diameter and volume models are useful tools for monitoring the development of forest restoration stands. In this study, equations of total height $(h)$ and total volume with bark $(v)$ were fitted as a function of the diameter at the breast height. The data were collected in 20 trees measured and scaled in mixed young forest stands of restoration located in the municipalities of Itapoã do Oeste and Cujubim, North of Rondônia State, in which five heightdiameter and volume regression models were tested. Also, the use of a mean form factor for volume estimation was examined. The models were judged by the coefficient of determination $\left(R^{2}\right)$, standard error of the estimate in percentage (Syx\%) and graphic residual analysis. All the tested height-diameter models presented satisfactory results, with Syx\% of $15 \%, R^{2}$ between 0.5443 and 0.5946 , being Henriksen's model was the best. The volume models presented Syx values of $11 \%, \mathrm{R}^{2}$ between 0.8497 and 0.9117 , therefore, precise. The best volume model was the Kopezky \& Gehrardt. It was concluded that there is a strong correlation between the mensuration variables (between 0.7573 e 0.9531 ), that the form factor is not accurate to estimate volume. In addition, the regression models, both height-diameter and volume, are accurate and can provide reliable estimates for monitoring young restoration stands in Amazon.

KEYWORDS: Volume Scaling, Young stands, Volume quantification. 


\section{INTRODUÇÃO}

Atualmente, a área desmatada na Amazônia totaliza 78 milhões de hectares, o que corresponde a 19,5\% desse bioma (INPE, 2017). Parte expressiva da Amazônia está em processo de degradação por exploração não sustentável de madeira e por outras ações antrópicas, principalmente nas áreas de fronteira agrícola (BECKER, 2007), conhecidas como Arco do Desmatamento, sendo responsável por $80 \%$ dos desmatamentos na região (FEARNSIDE, 2009).

Segundo INPE (2017), o estado de Rondônia apresenta cerca de $35 \%$ de seu território desmatado, o que corresponde a $83,5 \mathrm{mil} \mathrm{km}^{2}$. Dentre os vetores de desmatamento, destacam-se a pecuária bovina, praticada de forma extensiva há décadas no estado (BASTOS et al., 2015), e a agricultura mecanizada de larga escala, que avança sobre áreas consolidadas, promovendo novas frentes de desmatamento (COSTA et al., 2017) e alterando a paisagem.

Parte dos desmatamentos encontram-se em ambientes ciliares, consideradas Áreas de Preservação Permanente (APP) pela legislação em vigor (BRASIL, 2012), por serem ambientalmente frágeis (LIMA; ZAIKA, 2000) e possuírem funções de prestação de serviços ecossistêmicos fundamentais para sociedade (SALATI, 2001; FEARNSIDE, 2003).

Com a mudança no Código Florestal e outras normativas a fins, muitas áreas desmatadas foram abandonadas e submetidas ao processo de regeneração natural. Contudo, o uso prévio do solo por pastagens, notadamente ocupadas por Urochloa spp. (capimbraquiária) impõe dificuldades no processo de reconversão para floresta, o que pode seguir diferentes ritmos a depender das características da área e do seu uso prévio (NORDEN et al., 2011; MASSOCA et al., 2012; WILLIAMSON et al., 2012; ROCHA et al., 2016).

O plantio com espécies autóctones é uma alternativa para viabilizar e acelerar o processo de regeneração de tais áreas. Esse tem sido um dos métodos de restauração mais aplicados no Brasil, considerado de maior eficácia por facilitar e acelerar o processo de regeneração (LAMB, 2005; NICHOLS et al., 2001; CAMPOE et al., 2014).

Existem muitas experiências sobre restauração florestal nas regiões tropicais em várias partes do mundo, inclusive no Brasil, com muitos pesquisadores a se dedicar a desenvolver protocolos de monitoramento que ajudam a avaliar a performance dos plantios realizados (LE et al., 2012; ELLIOTT et al., 2013; WORTLEY et al., 2013; GONZÁLEZ et al., 2015; VIANI et al., 2017).

Tais trabalhos se baseiam em inventários realizados na vegetação, os quais usam estimativas indiretas que demandam, a rigor, a aplicação de equações hipsométricas e volumétricas. Tais equações permitem obter estimativas do volume e da biomassa vegetal produzida durante o curso da regeneração da floresta a partir de uma variável dendrométrica de fácil mensuração, como o diâmetro a 1,3 $m$ do solo $(d)$.

A maioria das equações hipsométricas e volumétricas encontradas na literatura brasileira foram ajustadas para espécies comerciais de rápido crescimento, principalmente Pinus sp., Eucalyptus sp., Acacia sp. e Tectona grandis L. (COUTO et al., 1987; MACHADO et al., 1994; FIGUEIREDO et al., 2006; SOUZA et al., 2008; AZEVEDO et al., 2011; MIGUEL et al., 2011; YOSHITANI JUNIOR et al., 2012). Por outro lado, poucas equações foram desenvolvidas para espécies nativas amazônicas, particularmente em plantios mistos de restauração.

Assim, este estudo tem como objetivo ajustar equações hipsométricas e volumétricas para povoamentos mistos de espécies nativas da Floresta Amazônica em Rondônia, a fim de subsidiar a avaliação do desempenho dos mesmos e contribuir para protocolos de monitoramento.

\section{MATERIAL E MÉTODOS}

Este trabalho foi realizado com dados oriundos de plantios de restauração florestal localizados nos municípios de Itapuã do Oeste e Cujubim, Norte do estado de Rondônia. Esses municípios estão inseridos na unidade estrutural cratônica (QUADROS et al., 2011) Sul-Amazônica da plataforma Sul-Americana, em região estabilizada geotectonicamente no Neoprotezoroico (PIRES, 2009).

Dados geológicos de Scandolara et al. (1999) evidenciam que a área está assentada em sua totalidade sobre rochas Paleoproterozoicas do complexo Jamari. Além disso, também possui áreas com formações Neoproterozoicas dos granitos jovens de Rondônia, com maior evidência na parte sudeste da área. Também podese notar a presença de coberturas constituídas por sedimentos indiferenciados Cenozoicos (QUADROS, 2010).

Foram identificadas cotas que variam entre 100 e $300 \mathrm{~m}$ e notabilizam-se pela ocorrência de extensas áreas aplainadas, levemente entalhadas pela rede de drenagem (RADAMBRASIL, 1978), estando frequentemente recobertas por coberturas detritolateríticas parcialmente desnudadas, gerando baixos platôs lateríticos (ADAMY, 2002).

A temperatura média anual encontra-se entre 24 a $26^{\circ} \mathrm{C}$ e a precipitação varia de 2.400 a $2.600 \mathrm{~mm}$ por ano. (GAMA, 2002). Os solos da região são classificados como 
Latossolos Amarelos Distróficos (RADAMBRASIL, 1978; RONDÔNIA, 2002), com característica de escassa fertilidade, devido à deficiência de nutrientes como fósforo $(\mathrm{P})$, potássio (K) e cálcio (Ca) (FORTUNA, 1988).

A Floresta Ombrófila Aberta Submontana é a fitofisionomia mais representativa (RADAMBRASIL, 1978; SILVA; VINHA, 2002), ocupando 95\% da unidade (BRASIL, 2005). Essa formação está diretamente relacionada com solos de característica quartzosa formados no Quaternário (RONDÔNIA, 2002).

Os povoamentos de restauração analisados estão situados em ambientes ciliares ao longo de corpos d'água de propriedades privadas, as quais encontravam-se desflorestadas e utilizadas como pastagem a mais de 10 anos. Os plantios mistos com espécies nativas foram implantados em dezembro de 2010 e janeiro de 2011, tendo aproximadamente 75 meses de idade na ocasião do término dos levantamentos objeto desta pesquisa.

\section{Coleta de dados}

Dois inventários foram realizados em julho de 2016 e em abril de 2017. Em cada ocasião, foram instaladas e mensuradas 10 parcelas permanentes, com dimensões de $20 \mathrm{~m} \times 10 \mathrm{~m}$, sendo identificados botanicamente todos os indivíduos plantados e medidos as circunferências a 1,3 m do solo e altura total (h). A identificação botânica das espécies vegetais ficou a cargo da Universidade Federal de Rondônia e do Museu Paraense Museu Emilio Goeldi.

$\mathrm{Na}$ proximidade de cada parcela, foi derrubada e mensurada uma árvore da espécie mais representativa dentro da unidade amostral. Assim, vinte indivíduos de diferentes espécies foram escolhidos para cubagem: Anacardium sp., Apuleia leiocarpa, Cassia grandis, Cecropia giganteum, Cedrela odorata, Ceiba pentandra, Cordia alliodora, Dipteryx odorata, Enterolobium schomburgkii, Enterolobium sp., Handroanthus sp., Hevea brasiliensis, Hymenaea courbaril, Inga cylindrica, Parkia multijuga, Schizolobium amazonicum e Stryphnodendron sp.

Foram realizadas diretamente medições individuais das variáveis $d, h$ e da altura comercial $(h c)$, situada no ponto de inversão morfológica. A cubagem rigorosa do volume total com casca $(v)$ foi realizada pelo do método de seções relativas de Hohenadl (SANQUETTA et al., 2014), com seções de $10 \%$ da $h c$. Os dados dendrométricos obtidos encontram-se na Tabela 1.
Tabela 1. Dados dendrométricos das 20 árvores cubadas em povoamentos jovens de restauração florestal no Norte de Rondônia, Brasil

\begin{tabular}{|c|c|c|c|c|}
\hline Espécie & $d(\mathrm{~cm})$ & $h(\mathrm{~m})$ & $h c(\mathrm{~m})$ & $v\left(\mathrm{~m}^{3}\right)$ \\
\hline Stryphnodendron sp. & 10,50 & 8,80 & 3,86 & 0,0322 \\
\hline Hymenolobium pulcherrimum & 6,05 & 6,15 & 2,90 & 0,0116 \\
\hline Inga cylindrica & 10,98 & 8,60 & 5,20 & 0,0289 \\
\hline Enterolobium sp. & 5,89 & 5,80 & 2,00 & 0,0145 \\
\hline Handroanthus sp. & 12,10 & 8,00 & 5,26 & 0,0338 \\
\hline Schizolobium amazonicum & 12,73 & 10,80 & 7,70 & 0,0353 \\
\hline Parkia multijuga & 11,94 & 7,70 & 5,45 & 0,0296 \\
\hline Hymenaea courbaril & 13,05 & 9,80 & 6,75 & 0,0371 \\
\hline Anarcadium sp. & 9,87 & 8,90 & 6,35 & 0,0208 \\
\hline Enterolobium schomburgkii & 5,73 & 6,30 & 3,25 & 0,0101 \\
\hline Hevea brasiliensis & 8,12 & 9,35 & 5,35 & 0,0209 \\
\hline Schizolobium amazonicum & 7,42 & 7,35 & 4,25 & 0,0140 \\
\hline Cedrela odorata & 7,51 & 6,65 & 6,65 & 0,0119 \\
\hline Hymenaea courbaril & 5,44 & 5,23 & 2,50 & 0,0124 \\
\hline Apuleia leiocarpa & 9,90 & 9,50 & 5,00 & 0,0253 \\
\hline Handroanthus sp. & 12,29 & 8,46 & 4,34 & 0,0304 \\
\hline Cordia alliodora & 12,19 & 10,97 & 10,02 & 0,0280 \\
\hline Dipteryx odorata & 7,48 & 6,55 & 2,82 & 0,0178 \\
\hline Ceiba pentandra & 10,66 & 6,25 & 4,71 & 0,0241 \\
\hline Cecropia giganteum & 12,41 & 11,80 & 8,80 & 0,0338 \\
\hline
\end{tabular}

\section{Análises estatísticas dos dados}

Os dados dendrométricos foram analisados por meio da correlação de Pearson $(r)$, ao nível de $5 \%$ de significância. Ademais, cinco modelos de regressão linear foram testados para a estimativa da altura total em função do diâmetro a 1,3 m do solo (Tabela 2).

Tabela 2. Modelos hipsométricos testados para 20 árvores mensuradas em povoamentos jovens de restauração florestal no Norte de Rondônia

\begin{tabular}{cc}
\hline Denominação & Modelo \\
\hline Linear & $\hat{y}_{i}=b_{0}+b_{1} d_{i}$ \\
Henriksen & $\hat{y}_{i}=b_{0}+b_{1} \ln \left(d_{i}\right)$ \\
Trorey & $\hat{y}_{i}=b_{0}+b_{1} d_{i}+b_{2} \ln \left(d_{i}{ }^{2}\right)$ \\
Stoffels \& van Soest & $\ln \left(\hat{y}_{i}\right)=b_{0}+b_{1} \ln \left(d_{i}\right)$ \\
Curtis & $\ln \left(\hat{y}_{i}\right)=b_{0}+b_{1}\left(1 / d_{i}\right)$ \\
\hline
\end{tabular}

In = logaritmo neperiano; $\hat{y}_{i}=$ altura estimada $(\mathrm{m}) ; b_{0}, b_{1}$ e $b_{2}=$ coeficientes de regressão; e $d_{i}=$ diâmetro a $1,3 \mathrm{~m}$ do solo $(\mathrm{cm})$.

Para a estimativa do volume, foram testadas duas técnicas:

1) Fator de forma artificial médio; e

2) Modelos volumétricos. 
Fator de forma artificial médio

O fator de forma médio artificial ( $\bar{f}$ ) foi obtido por meio do cálculo da média geral correspondente às 20 árvores, conforme a equação (1):

$$
\bar{f}=\frac{\sum_{i=1}^{n}\left(\frac{v_{i}}{v c_{i}}\right)}{n}
$$

Em que:

$v_{i}=$ volume rigoroso obtido através de cubagem $\left(\mathrm{m}^{3}\right)$; e $v c_{i}=$ volume do cilindro calculado a partir do $d\left(\mathrm{~m}^{3}\right)$.

A estimativa do volume individual foi obtida por meio da multiplicação do volume do cilindro pelo fator de forma médio, conforme a equação (2).

$$
\hat{v}_{i}=\frac{\pi d_{i}^{2}}{40.000} \cdot h_{i} \cdot \bar{f}
$$

Em que:

$\hat{v}_{i}=$ volume estimado $\left(\mathrm{m}^{3}\right)$;

$d_{i}=$ diâmetro a $1,3 \mathrm{~m}$ de altura $(\mathrm{cm}) ; \mathrm{e}$

$h_{i}=\operatorname{altura}$ total $(\mathrm{m})$.

\section{Modelos volumétricos}

Foram testados cinco modelos volumétricos (Tabela 3) com o emprego do método dos mínimos quadrados ordinários por regressão linear, sendo três modelos de simples entrada $(d)$ e dois de dupla entrada $(d$ e $h)$.

Tabela 3. Modelos volumétricos testados para 20 árvores mensuradas em povoamentos jovens de restauração florestal no Norte de Rondônia

\begin{tabular}{cc}
\hline Denominação & Modelo \\
\hline Linear & $\hat{y}_{i}=b_{0}+b_{1} d_{i}$ \\
Kopezky \& Gehrardt & $\hat{y}_{i}=b_{0}+b_{1} d_{i}{ }^{2}$ \\
Husch & $\ln \left(\hat{y}_{i}\right)=b_{0}+b_{1} \ln \left(d_{i}\right)$ \\
Spurr & $\hat{y}_{i}=b_{0}+b_{1}\left(d_{i}{ }^{2} h_{i}\right)$ \\
Schumacher \& Hall & $\ln \left(\hat{y}_{i}\right)=b_{0}+b_{1} \ln \left(d_{i}\right)+b_{2} \ln \left(h_{i}\right)$ \\
\hline
\end{tabular}

A correção das estatísticas de ajuste para os modelos de natureza logarítmica foi realizada pelo Fator de Correção de Meyer (FCM), conforme a equação (3).

$$
F C M=e^{\left(0,5 S y x^{2}\right)}
$$

Em que:

FCM = Fator de Correção de Meyer; e

Syx = erro padrão da estimativa.
Tanto os modelos hipsométricos quanto os volumétricos foram avaliados pelo coeficiente de determinação ajustado ( $R^{2}$ aj.), erro padrão da estimativa percentual (Syx\%) e análise gráfica dos resíduos (Tabela 4).

Tabela 4. Critérios de seleção dos ajustes hipsométricos e volumétricos para 20 árvores mensuradas nos povoamentos jovens de restauração florestal no Norte de Rondônia

\begin{tabular}{cc}
\hline Critério & Formulação \\
\hline $\begin{array}{c}\text { Coeficiente de } \\
\text { determinação ajustado }\end{array}$ & $R^{2}{ }_{a j .}=1-\frac{(n-1)}{(n-k)}\left(1-R^{2}\right) \quad$ (5) \\
$\begin{array}{c}\text { Erro padrão da } \\
\text { estimativa percentual }\end{array}$ & Syx\% $=\frac{\sqrt{\frac{\sum_{i-1}^{n} e_{i}^{2}}{n-k}}}{\bar{y}} 100$ \\
Resíduos \% & $e_{i} \%=\frac{\left(y_{i}-\hat{y}_{i}\right)}{y_{i}} 100$
\end{tabular}

$n$ = número de observações; $k=$ número de parâmetros do modelo; e $\bar{v}=$ volume observado médio $\left(\mathrm{m}^{3}\right)$.

\section{RESULTADOS E DISCUSSÃO}

\section{Correlação das variáveis}

Na análise de correlação, observou-se que a altura total (h) apresentou forte e significativa correlação $(r=0,7686)$ com o diâmetro a $1,3 \mathrm{~m}$ do solo $(d)$, o que viabiliza o desenvolvimento de equações hipsométricas para os dados em questão. As correlações de $d$ e $h$ com o volume (v) foram fortes, sendo equivalentes a 0,9531 e 0,7573, respectivamente, e significativas a $95 \%$ de probabilidade. Por conseguinte, espera-se que as equações ajustadas proporcionem resultados precisos para aplicá-las em situações práticas de monitoramento de povoamentos de restauração florestal na região.

\section{Estimativas hipsométricas}

Os ajustes das equações hipsométricas apresentaram $\mathrm{R}^{2}$ aj. inferiores a 0,6 , o que pode ser considerado como baixo grau de ajuste, quando comparados a outros trabalhos (SILVA et al., 1984; TONINI et al., 2005; IMAÑAENCINAS et al., 2009; RÉ et al., 2015). Porém, os valores de Syx\% foram todos próximos a 15\% (Tabela 5), o que denota que os ajustes são precisos. Fato também corroborado pela análise gráfica de resíduos (Figura 1).

O modelo de Henriksen apresentou o menor Syx\%, sendo, portanto, o de melhor desempenho, com erro da ordem de 1,85 m. Entretanto, pouca diferença foi notada em relação aos demais modelos testados, exceto o de 
Trorey, que apresentou maior valor de Syx\% e menor $\mathrm{R}^{2}$. Aragão et al. (2015) também observaram que o modelo de Henriksen foi o de melhor desempenho para plantios puros de Pterogyne nitens.

Tabela 5. Estatísticas e coeficientes de regressão para modelos hipsométricos testados para 20 árvores mensuradas em povoamentos jovens de restauração florestal no Norte de Rondônia

\begin{tabular}{cccccc}
\hline Modelo & $\boldsymbol{b}_{\boldsymbol{0}}$ & $\boldsymbol{b}_{\mathbf{1}}$ & $\boldsymbol{b}_{\mathbf{2}}$ & $\mathbf{R}^{\mathbf{2} a j .}$ & Syx\% \\
\hline Linear & 2,94503 & 0,54125 & & 0,5908 & 15,07 \\
Henriksen & $-2,47873^{\text {ns }}$ & 4,78151 & & 0,5946 & 15,00 \\
$\begin{array}{c}\text { Trorey } \\
\text { Stoffels \& }\end{array}$ & $1,76411^{\text {ns }}$ & $0,81902^{\text {ns }}$ & $-0,01503^{\text {ns }}$ & 0,5443 & 15,48 \\
van Soest & 0,71044 & 0,61029 & & 0,5933 & 15,02 \\
Curtis & 2,65239 & $-5,11724$ & & 0,5923 & 15,04 \\
\hline
\end{tabular}

ns = não significativo estatisticamente a $95 \%$ de probabilidade.

Em florestas nativas, bem como, em plantios mistos, é de se esperar maior heterogeneidade no desenvolvimento das árvores (RÉ et al., 2015), contribuindo para maiores valores de erro padrão da estimativa (ZANON et al., 1996; COLPINI et al., 2009).

Observando a análise de resíduos dos modelos hipsométricos testados (Figura 1), percebe-se que todos os modelos se ajustaram aos dados, não ocorrendo problemas de tendência como valores extremos e autocorrelação. Também foi observado que os erros de estimativa dos modelos hipsométricos se concentraram entre $\pm 50 \%$.

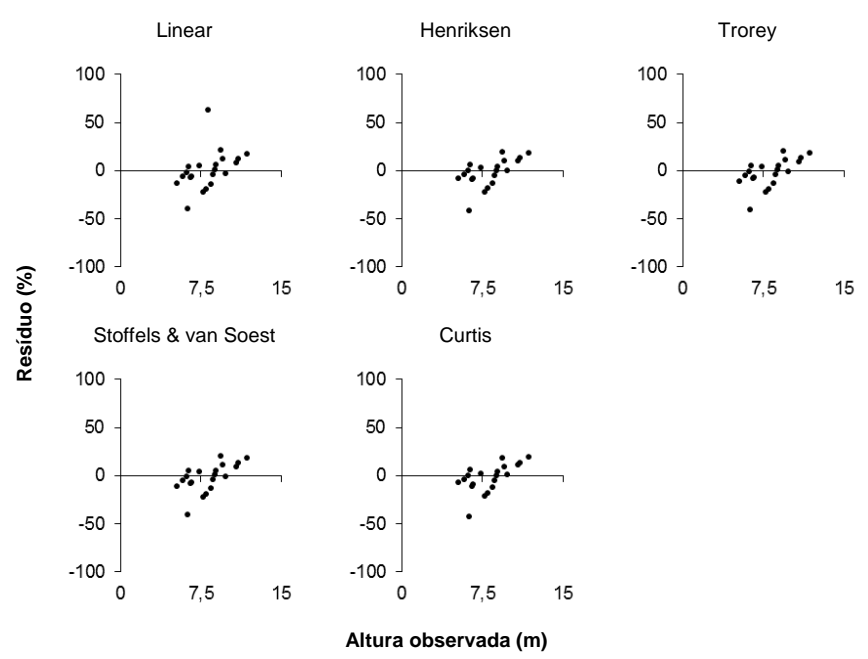

Figura 1. Resíduos das estimativas de altura para 20 árvores mensuradas em povoamentos jovens de restauração florestal no Norte de Rondônia.

\section{Estimativas volumétricas}

Fator de forma artificial médio

O fator de forma artificial médio observado foi de 0,4488 , com desvio padrão de $\pm 0,2160$ e coeficiente de variação de $48,12 \%$. Notou-se que, após as estimativas volumétricas individuais, o uso do fator de forma médio acarretou em erro elevado, da ordem de 58,88\%, não sendo preciso e acurado para as estimativas volumétricas, devendo ser descartada a hipótese de aplicá-lo na prática. Schröder et al. (2013), em seu trabalho para plantios homogêneos, observou tal constatação.

\section{Modelos volumétricos}

Observou-se que os modelos apresentaram $R^{2} a j$. superiores a 0,90, exceto para o modelo de Spurr (Tabela 6). Ademais, os valores de Syx\% variaram de $11,65 \%$ a $11,87 \%$, sendo considerados satisfatórios. Novamente, o modelo de Spurr apresentou as piores estatísticas, com Syx\% de 15,21\%. Esses valores são considerados baixos, quando comparados a outros trabalhos (SILVA et al., 1984; TONINI et al., 2005; IMAÑA-ENCINAS et al., 2009; RÉ et al., 2015).

Tabela 6. Estatísticas de ajuste e coeficientes de regressão para modelos volumétricos testados para 20 árvores mensuradas em povoamentos jovens de restauração florestal no Norte de Rondônia

\begin{tabular}{cccccc}
\hline Modelo & $\boldsymbol{b}_{\mathbf{0}}$ & $\boldsymbol{b}_{\mathbf{1}}$ & $\boldsymbol{b}_{\mathbf{2}}$ & $\mathbf{R}^{\mathbf{2}} \mathbf{a j .}$ & Syx\% \\
\hline Linear & $-0,00752$ & 0,00324 & - & 0,9084 & 11,87 \\
$\begin{array}{c}\text { Kopezky \& } \\
\text { Gehrardt }\end{array}$ & 0,00630 & 0,00017 & - & 0,9117 & 11,65 \\
Husch & $-6,83916$ & 1,35549 & - & 0,9117 & 11,66 \\
Spurr & 0,01054 & 0,00002 & - & 0,8497 & 15,21 \\
$\begin{array}{c}\text { Schumacher } \\
\text { \& Hall }\end{array}$ & $-6,90579$ & 1,29802 & $0,09378^{\text {ns }}$ & 0,9035 & 11,86 \\
\hline ns = não significativo estatisticamente a 95\% de probabilidade.
\end{tabular}

Diante dos resultados obtidos, pode-se inferir que os modelos de regressão se mostraram satisfatórios para a estimativa do volume individual. Contudo, devido ao desempenho inferior, quando avaliadas as estatísticas de ajuste, o modelo de Spurr foi considerado não satisfatório. De maneira geral, os modelos de Kopezky \& Gehrardt e de Husch apresentam as melhores performances, com leve vantagem para o primeiro.

Entretanto, deve-se considerar alguns aspectos 
práticos, como a natureza logarítmica e a necessidade de correção da discrepância logarítmica. Nesse contexto, o modelo de Husch foi considerado vantajoso pela simplicidade e robustez, uma vez que o modelo de Kopezky $\&$ Gehrardt é quadrático no $d$, o que pode agravar os erros de estimativa fora do intervalo dos dados utilizados no ajuste.

Foi empregada a análise gráfica dos resíduos, a qual confirmou que a técnica do fator de forma médio apresenta tendenciosidade em subestimar o volume para as árvores com menores diâmetros e superestimar para as de maiores diâmetros. Entretanto, os dois melhores modelos de regressão não apresentaram tendências em seu ajuste e tiveram comportamento semelhante em relação à distribuição dos resíduos (Figura 2).

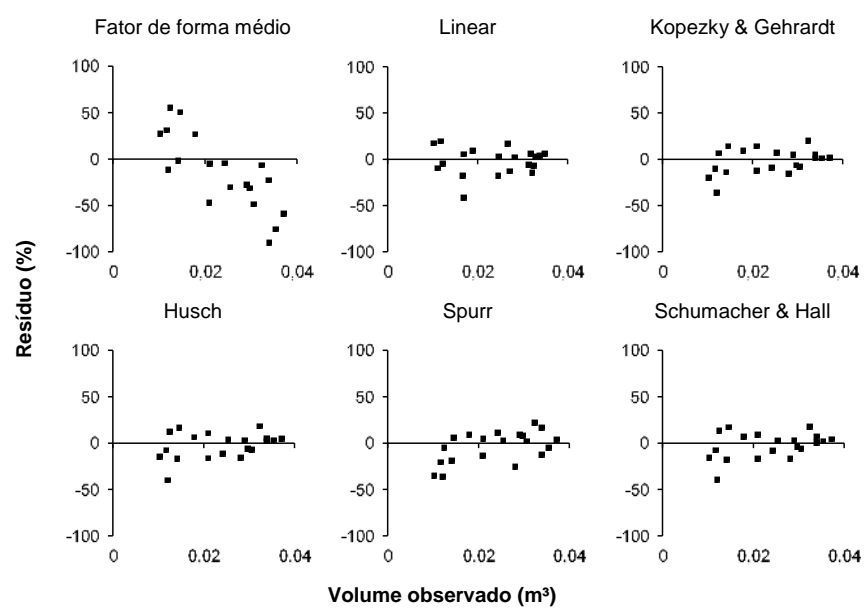

Figura 2. Resíduos das estimativas de volume para 20 árvores mensuradas em povoamentos jovens de restauração florestal no Norte de Rondônia.

Pode-se afirmar que os modelos de dupla entrada não apresentaram melhores estimativas, em comparação aos de simples entrada. A partir das estatísticas de ajuste, notou-se que o modelo de Spurr apresentou os piores valores, enquanto o coeficiente $b_{2}$ do modelo de Schumacher \& Hall, referente à altura, não foi significativo estatisticamente. Esse fato pode ser explicado pela menor correlação entre a altura e o volume, conforme descrito anteriormente.

Em atividades de inventário florestal, é comum medir todos os diâmetros das árvores contidas nas unidades amostrais e parte das alturas, pois a mesma é considerada mais complexa de ser mensurada (HUSCH et al., 2003; CRECENTE-CAMPO et al., 2009). Assim, diagnosticar a contribuição da altura em estimativas volumétricas é importante, uma vez que o volume é a variável de maior interesse, pois expressa o estoque de uma floresta.
Não existem muitos estudos morfométricos de restauração em florestas brasileiras. A maioria dos trabalhos com esse enfoque referem-se as plantações florestais comerciais com espécies de rápido crescimento e, em menor escala, de florestas nativas regeneradas naturalmente (MIRANDA, 2008).

Contudo, as diferentes arquiteturas das árvores em povoamentos de restauração, especialmente os jovens, em relação às plantações comerciais e às florestas nativas maduras, pode ser um fator de indução aos erros de estimativas da altura e do volume e, por conseguinte, dos erros de avaliação quanto à performance da atividade de restauração florestal.

Modelos de relações hipsométricas e volumétricas foram testados por Ré et al. (2015) para nove espécies em sistemas de restauração na Floresta Estacional Semidecidual da Mata Atlântica no estado de São Paulo, os quais concluíram que os modelos atenderam às expectativas. Os autores comentaram também que as equações podem ser livremente empregadas nos modelos alternativos de restauração estudados, sendo úteis para o manejo e a conservação das áreas em estudo.

Modelos de relação hipsométrica em florestas nativas mistas do Brasil foram estudados por Sanquetta et al. (2013), ao realizarem estudos para espécies da Floresta Ombrófila Mista no estado do Paraná. Apesar da grande dispersão encontrada nas relações hipsométricas, as equações analisadas pelos autores mostraram-se acuradas, apresentando melhorias quando empregada a estratificação por família botânica.

Adicionalmente, Hess et al. (2014) realizaram estudos na Floresta Amazônica, com enfoque na relação hipsométrica de espécies comerciais. Esses autores também observaram grande dispersão nos dados e, apesar de terem encontrado indicadores estatísticos satisfatórios, sugeriram cautela na aplicação das equações, devido à possibilidade de erros na estimativa.

Na Amazônia Brasileira, utiliza-se o fator de forma de 0,7 para estimativa de volume de fustes, ocasionando sérios erros de estimativas volumétricas (FIGUEIREDO et al., 2009). O fator de forma calculado neste estudo $(0,448)$ foi muito inferior ao valor referencial empregado em muitos trabalhos sobre quantificação volumétrica em espécies nativas da Amazônia.

Atualmente, existem na Amazônia esforços para obter estimativas robustas e precisas do volume de espécies florestais, incluindo-se o aprimoramento das técnicas de regressão e do uso de inteligência artificial como meio de melhorar as primeiras estimativas realizadas a partir da década de 1980 (CYSNEIROS, 2016). 
Algumas questões básicas ainda não foram resolvidas na estimativa do volume na região Amazônica, uma vez que alguns autores defendem que a altura não pode ser mensurada corretamente devido à dificuldade de visualização da copa, o que justifica o emprego de equações volumétricas de simples entrada.

No estudo de Gimenez (2013), evidenciou-se que as equações de volume de simples entrada, com o diâmetro a 1,3 $\mathrm{m}$ do solo como variável independente, resultam em estimativas mais precisas em comparação com as de dupla entrada. Essa abordagem metodológica pode reduzir a necessidade da medição da altura, a qual é mais sujeita a erros e demanda mais tempo e recursos.

\section{CONCLUSÕES}

Os modelos hipsométricos ajustados resultam em estatísticas satisfatórias, podendo ser utilizados para estimar a altura total das árvores em função do diâmetro a 1,3 $\mathrm{m}$ do solo, em que o modelo de Henriksen é o selecionado como melhor entre os testados.

O fator de forma médio, igual a 0,448 , não é preciso para estimar volume do fuste, sendo inferior ao valor de 0,7 comumente utilizado para espécies florestais da Amazônia.

Os modelos volumétricos ajustados resultam em estatísticas satisfatórias, podendo ser utilizados para estimar o volume do fuste com casca em função do diâmetro a 1,3 $\mathrm{m}$ do solo e da altura total das árvores.

Os modelos de simples entrada, que utilizam somente do diâmetro a 1,3 $\mathrm{m}$ do solo como variável independente de estimação, propiciam melhores resultados comparativamente aos de dupla entrada, em que o modelo de Husch é o selecionado como melhor entre os testados.

Os modelos hipsométricos e volumétricos selecionados são precisos e podem gerar estimativas confiáveis para o monitoramento de povoamentos jovens de restauração florestal na Amazônia.

\section{REFERÊNCIAS}

ADAMY, A. Estudos das formas de relevo. In: Atlas Geoambiental de Rondônia. Porto Velho: SEDAM, v. 2, p. 3-6, 2002.

ARAGÃO, M. A.; BARRETO, P. A. B.; PAULA, A.; CARVALHO, F. F.; FRAGA, M. P. Modelos de altura para Pterogyne nitens Tul. em plantio puro no sudoeste da Bahia. Enciclopédia Biosfera, v. 11, n. 21, p. 1340-1351, 2015.

AZEVEDO, T. L.; MELLO, A. A.; FERREIRA, R. A.; SANQUETTA, C. R.; NAKAJIMA, N. Y. Equação hipsométricas e volumétricas para um povoamento de Eucalyptus sp. localizado na FLONA do Ibura, Sergipe. Revista Brasileira de Ciência Agrária, v. 6, n. 1, p. 105-
112, 2011.

BASTOS, A. S; MANIESI, V.; GOMES, F. B. Vulnerabilidade natural à erosão no Sudoeste da Amazônia associada aos seus modos de ocupação? O caso do entorno da Terra Indígena Uru Eu Wau Wau. Revista Brasileira de Geomorfologia, v. 16, p. 271-282, 2015.

BECKER, B. Amazônia: geopolítica na virada do III milênio. Rio de Janeiro: Garamound, 2007. 180 p.

BRASIL. Plano de manejo da Floresta Nacional do Jamari. Brasília: IBAMA, v. 1, 2005. 158 p.

BRASIL. Lei no 12.651 de 25 de maio de 2012. Brasília: Diário Oficial da União, 2012. Disponível em: <http://www.planalto.gov.br/ccivil_03/_ato2011-014/2012/lei/ |12651.htm>. Acesso em: 21/07/2017.

CAMPOE, O. C.; IANNELLI, C.; STAPE, J. L.; COOK, R. L.; MENDES, J. C. T.; VIVIAN, R. Atlantic forest tree species responses to silvicultural practices in a degraded pasture restoration plantation: from leaf physiology to survival and initial growth. Forest Ecology and Management, v. 313, n. 1, p. 233-242, 2014.

COLPINI, C.; TRAVAGINI, P. D.; SOARES, S. T. Determinação do volume, do fator de forma e da porcentagem de casca de árvores individuais em uma floresta ombrófila aberta na região noroeste de Mato Grosso. Acta Amazônica, v. 39, n. 1, p. 97-104, 2009.

COSTA, O. B.; MATRICARDI, E. A. T.; PEDLOWSKI, M. A.; COCHRANE, M. A.; FERNANDES, L. C. Spatiotemporal mapping of soybean plantations in Rondônia, Western Brazilian Amazon. Acta Amazônica, v. 47, n. 1, p. 29-38, 2017.

COSTA, O. B.; MATRICARDI, E. A. T.; PIRES, J. S. R. Análise do processo de fragmentação da floresta nos municípios de Corumbiara e Buritis - RO. Floresta e Ambiente, v. 22, n. 3, p. 334344, 2015.

COUTO, H. T. Z.; BASTOS, N. L. M.; Modelos de equações de volume e relações hipsométricas para plantações de Eucalyptus no Estado de São Paulo. IPEF, n. 37, p. 33-44, 1987.

CRECENTE-CAMPO, F.; TOMÉ, M.; SOARES, P.; DIÉGUEZ-ARANDA, $U$. A generalized nonlinear mixed-effects height-diameter model for Eucalyptus globulus L. in northwestern Spain. Forest Ecology and Management, v. 259, n. 1, p. 943-952, 2010.

CYSNEIROS, V. C. Estratégias para modelagem do volume comercial em florestas tropicais. 117 f. 2016. Dissertação (Mestrado) - Universidade Federal do Paraná, Curitiba.

ELLIOTT, S.; BLAKESLEY, D.; HARDWICK, K. Restoring tropical forests: a practical guide. London. 2013. $360 \mathrm{p}$.

FEARNSIDE, P. M. A floresta amazônica nas mudanças globais. Manaus: Instituto Nacional de pesquisas da Amazônia, 2003. 134 p.

FEARNSIDE, P. M. Aquecimento global na Amazônia: impactos e mitigação. Acta Amazonica, v. 39, n. 4, p. 1003-1012, 2009.

FIGUEIREDO, E. O.; SCHROEDER, R.; PAPA, D. A. Fatores de forma para 20 espécies florestais comerciais da Amazônia. Rio Branco: 
Empresa Brasileira de Pesquisa Agropecuária. Comunicado técnico, n. 173, 2009. 4 p.

FIGUEIREDO, E. O.; SCOLFORO, J. R. S.; OLIVEIRA, A. D. Seleção de modelos polinomiais para representar o perfil e volume do fuste de Tectona grandis L.f. Acta amazônica, v. 36, n. 4, p. 465-482, 2006.

FORTUNA, A. Caracterização dos trópicos úmidos brasileiros. In: Hébette, J. (coord). Natureza, tecnologia e sociedades. Belém: NAEA, p. 7-13, 1988.

GAMA, M. J. Clima. In: Atlas geoambiental de Rondônia. Porto Velho: SEDAM, 2002.

GIMENEZ, B. O. Equações volumétricas e o modelo que utiliza o fator de forma médio: um estudo de caso na Amazônia Central. 62 f. 2013. Dissertação (Ciências de Florestas Tropicais) - Instituto Nacional de Pesquisas da Amazônia, Manaus.

GONZALEZ, E.; SHER, A. A.; TABACCHI, E.; MASIP, A.; POULIN, M. Restoration of riparian vegetation: a global review of implementation and evaluation approaches in the international, peer-reviewed literature. Journal of Environmental Management, v. 158, p. 85-94, 2015.

HESS, A. F.; BRAZ, E. M.; THAINES, F.; MATTOS, P. P. Ajuste de relação hipsométrica para espécies da Floresta Amazônica. Ambiência, v. 10, n. 1, p. 21-29, 2014.

HUSCH, B.; BEERS, T. W.; KERSHAW Jr., J. A. Forest Mensuration. 4 ed. Nova Jersey: John Wiley \& Sons, 2003. 456 p.

IMAÑA-ENCINAS, J.; SANTANA, O. A.; PAULA, J. E.; IMAÑA, C. R. Equações de volume de madeira para o cerrado de Planaltina de Goiás. Floresta, v. 39, n. 1, p. 107-116, 2009.

INPE - Instituto Nacional de Pesquisas Espaciais. Projeto PRODES - monitoramento da floresta amazônica por satélite. Disponível em: <http://www.obt.inpe.br/prodes/sisprodes2000_2016.htm> Acesso em: 24/07/2017.

LAMB, D. Restoration of degraded tropical forest landscapes. Science, v. 310, p. 1628-1632, 2005.

LE, H. D.; SMITH, C.; HERBOHN, J.; HARRISON, S. More than just trees: assessing reforestation 381 success in tropical developing countries. Journal of Rural Studies, v. 28, p. 5-19, 2012.

LIMA, W. P.; ZAKIA, M. J. B. Hidrologia de matas ciliares. In: Matas ciliares: conservação e recuperação. RODRIGUES, R. R.; LEITÃO FILHO, H. F. (Eds). São Paulo: Editora Universidade de São Paulo, p. $33-44,2000$.

MACHADO, S. A.; BAILEY, R. L.; BASSO, S. F.; JUNIOR, V. G. B. Análise do comportamento da relação hipsométrica com respeito à idade para plantações de Pinus elliotti no Estado do Paraná. Cerne, v. 1, n. 1, p. 05-12, 1994.

MIGUEL, E. P.; MACHADO, S. A.; FIGUEIREDO FILHO, A.; ARCE, J. E. Modelos polinomiais para representar o perfil e o volume do fuste de Eucalyptus urophylla na região norte do Estado de Goiás. Floresta, v. 41, n. 2, p. 355-368, 2011.
MASSOCA, P. E. S.; JACOVAK, A. C. C.; BENTOS, T. V.; WILLIAMSON, G. B.; MESQUITA, R. D. C. G. Dinâmica e trajetórias da sucessão secundária na Amazônia central. Boletim do Museu Paraense Emílio Goeldi - Ciências Naturais, v. 7, n. 3, p. 235-250, 2012.

MIRANDA, D. C. Modelos matemáticos de estoque de biomassa e carbono em áreas de restauração florestal no sudoeste paulista. 130 f. 2008. Dissertação (Mestrado em Engenharia Florestal) - Universidade Federal do Paraná, Curitiba.

NICHOLS, J. D.; ROSEMEYER, M. E.; CARPENTER, F. L.; KETTLER, J. Intercropping legume trees with native timber trees rapidly restores cover to eroded tropical pasture without fertilization. Forest Ecology and Management, v. 152, n. 1/3, p. 195-209, 2001.

NORDEN, N.; MESQUITA, R. C. G.; BENTOS, T. V.; CHAZDON, R. L.; WILLIAMSON, G. B. Contrasting community compensatory trends in alternative successional pathways in central Amazonia. Oikos, v. 120 , n. 1, p. 143-151, 2011.

PIRES, F. R. M. Arcabouço geológico. In: Geomorfologia do Brasil. CUNHA, S. B. C.; GUERRA, A. J. T. (Eds.), 5. ed. Rio de Janeiro: Bertrand Brasil, p. 17-69, 2009.

QUADROS, M. L. E. S.; PALMEIRA, L. C. M.; CASTRO, C. C. Geologia e recursos minerais da folha rio Machado (SC.20-X-C). Escala 1:250.000. Porto Velho: CPRM, 2011.

RADAM BRASIL. Levantamento de recursos naturais. Projeto RADAMBRASIL, Folha SC.20 - Porto Velho. DNPM, 1978.

RÉ, D. S.; ENGEL, V. L.; OTA, L. M. S.; JORGE, L. A. B. Equações alométricas em plantios mistos visando à restauração da floresta estacional semidecidual. Cerne, v. 21, n. 1, p. 133-140, 2015.

ROCHA, G. P. E.; VIEIRA, D. L. M.; SIMON, M. F. Fast natural regeneration in abandoned pastures in southern Amazonia. Forest Ecology and Management, v. 370, p. 93-101, 2016.

RONDÔNIA. As unidades de conservação de Rondônia. 2. ed. Porto Velho. 2002, 97 p.

SALATI, E. Mudanças climáticas e o ciclo hidrológico na Amazônia. In: Causas e Dinâmica do Desmatamento da Amazônia. Brasília: MMA, p. 153-172, 2001.

SANQUETTA, C. R.; CORTE, A. P. D.; RODRIGUES, A. L.; WATZLAWICK, L. F. Inventários florestais: planejamento e execução. Curitiba, 2014. 409 p.

SANQUeTtA, C. R.; CORTE, A. P. D.; ROGLIN, A.; PIMENTEL, A. Relações diâmetro-altura para espécies lenhosas em um fragmento de Floresta Ombrófila Mista no Sul do Paraná. Iheringia Série Botânica, v. 68, n. 1, p. 103-114, 2013.

SCANDOLARA, J. E.; RIZZOTO, G. J.; AMORIM, J. L.; BAHIA, R. C. B.; QUADROS, M. L.; SILVA, C. S. Mapa geológico de Rondônia na escala de 1:1.000.000, CPRM. Porto Velho, 1999.

SCHRÖDER, T.; PEREIRA, L. D.; HOFIÇO, N. S. A. Comparação de métodos de estimativa de volume total para Eucalyptus grandis W. Hill ex Maiden. Floresta e Ambiente, v. 20, n. 4, p. 480-486, 
2013.

SILVA, J. N. M.; CARVALHO, J. O. P.; LOPES, J. C. A.; CARVALHO, M. S. P. Equações de volume para a floresta nacional do Tapajós. Boletim de Pesquisa Florestal, v. 8/9, p. 50-63, 1984.

SILVA, R. B.; VINHA, E. Vegetação: biodiversidade de espécies florestais. In: Atlas geoambiental de Rondônia. Porto Velho: SEDAM, p. 96-101, 2002.

SOUZA, C. A. M.; SILVA, G. F.; XAVIER, A. C.; MENDONÇA, A. R.; ALMEIDA, A. Q. Avaliação de modelos de afilamento nãosegmentados na estimação da altura e volume comercial de Eucalyptus sp. Ciência Florestal, v. 18, n. 3, p. 393-405, 2008.

TONINI, H.; ARCO VERDE, M. F.; SÁ, S. P. P. Dendrometria de espécies nativas em plantios homogêneos no Estado de Roraima - Andiroba (Carapa guianensis Aubl), Castanhado-Brasil (Bertholletia excelsa Bonpl.), Ipê-roxo (Tabebuia avellanedae Lorentz ex Griseb) e Jatobá (Hymenaea courbaril L.). Acta Amazônica, v. 35, n. 3, p. 353-362, 2005.

VIANI, R. A. G.; HOLL, K. D.; PADOVEZI, A.; STRASSBURG, B. B. N.; FARAH, F. T.; GARCIA, L. C.; CHAVES, R. B.; RODRIGUES, R. R.; BRANCALIO, P. H. S. Protocol for monitoring tropical forest restoration: perspectives from the Atlantic forest restoration pact in Brazil. Tropical Conservation Science, v. 10, p. 1-8, 2017.

WILLIAMSON, G. B.; BENTOS, T. V.; LONGWORTH, J. B.; MESQUITA, R. C. G. Convergence and divergence in alternative successional pathways in Central Amazonia. Plant Ecology \& Diversity, v. 7, n. 1, p. 341-348, 2012.

WORTLEY, L.; HERO, J. M.; HOWES, M. Evaluating ecological restoration success: a review of the literature. Restoration Ecology, v. 5, p. 537-543, 2013.

YOSHITANI JUNIOR, M.; NAKAJIMA, N. Y.; ARCE, J. E.; MACHADO, S. A.; DRUSZCZ, J. P.; HOSOKAWA, R. T.; MELLO, A. A. Funções de afilamento para plantios desbastados de Pinus taeda. Floresta, $v$. 42, n. 1, p. 169-176, 2012.

ZANON, M. L. B.; FINGER, C. A. G.; SCHNEIDER, P. R.; KLEIN, J.; MEYER, E.; COELHO, M. C. B. Funções para descrever a relação altura diâmetro de Eucalyptus dunnii Maiden. Ciência Rural, v. 26, n. 1, p. 87-90, 1996. 